\title{
Angular distribution in the neutron-induced fission of actinides
}

L.S. Leong ${ }^{1}$, L. Tassan-Got ${ }^{1}$, D. Tarrio ${ }^{2}$, L. Audouin ${ }^{1}$, C. Paradela ${ }^{2}$, I. Duran ${ }^{2}$, C. Le Naour ${ }^{1}$,

S. Altstadt ${ }^{3}$, J. Andrzejewsky ${ }^{4}$, M. Barbagallo ${ }^{5}$, V. Bécares ${ }^{6}$, F. Bečváŕ ${ }^{7}$, F. Belloni ${ }^{8}$,

E. Berthoumieux ${ }^{8,9}$, J. Billowes ${ }^{10}$, V. Boccone ${ }^{9}$, D. Bosnar ${ }^{11}$, M. Brugger ${ }^{9}$, F. Calvin̄o ${ }^{12}$, M. Calviani ${ }^{9}$,

D. Cano-Ott ${ }^{6}$, C. Carrapiço ${ }^{13}$, F. Cerutti ${ }^{9}$, E.Chiaveri ${ }^{9}$, M. Chin ${ }^{9}$, N. Colonna ${ }^{5}$, G. Cortés ${ }^{12}$,

M.A. Cortés-Giraldo ${ }^{14}$, M. Diakaki ${ }^{15}$, C. Domingo-Pardo ${ }^{16}$, R. Dressler ${ }^{16}$, N. Dzysiuk ${ }^{17}$,

C. Eleftheriadis ${ }^{18}$, A. Ferrari ${ }^{9}$, K. Fraval ${ }^{8}$, S. Ganesan $^{19}$, A.R. García ${ }^{6}$, G. Giubrone ${ }^{16}$,

M.B. Gómez-Hornillos ${ }^{12}$, I.F. Gonçalves ${ }^{13}$, González-Romero ${ }^{6}$, E. Griesmayer ${ }^{20}$, C. Guerrero ${ }^{9}$,

F. Gunsing ${ }^{8}$, P. Gurusamy ${ }^{19}$, D.G. Jenkins ${ }^{21}$, E. Jericha ${ }^{20}$, E. Kadi ${ }^{9}$, F. Käppeler ${ }^{22}$, D. Karadimos ${ }^{15}$,

N. Kivel ${ }^{32}$, P. Koehler ${ }^{23}$, M. Kokkoris ${ }^{15}$, G. Korschinek ${ }^{33}$, J. Kroll ${ }^{7}$, M. Krtička ${ }^{7}$, C. Langer ${ }^{3}$,

C. Lederer ${ }^{3,24}$, H. Leeb ${ }^{20}$, R. Losito ${ }^{9}$, A. Manousos ${ }^{18}$, J. Marganiec ${ }^{4}$, C. Massimi ${ }^{25}$, T. Martínez ${ }^{6}$,

P.F. Mastinu ${ }^{17}$, M. Mastromarco ${ }^{5}$, M. Meaze ${ }^{5}$, A. Mengon ${ }^{26}$, E. Mendoza ${ }^{6}$, P.M. Milazzo ${ }^{27}$,

T. Mingrone ${ }^{6}$, M. Mirea ${ }^{28}$, W. Mondelaers ${ }^{29}$, A. Pavlik ${ }^{24}$, J. Perkowski ${ }^{4}$, M. Pignatari ${ }^{34}$, A. Plompen ${ }^{29}$,

J. Praena ${ }^{14}$, J.M. Quesada ${ }^{14}$, T. Rauscher ${ }^{30}$, R. Reifhart ${ }^{3}$, A. Riego ${ }^{12}$, F. Roman ${ }^{9,28}$, C. Rubbia ${ }^{9,31}$,

R.Sarmento ${ }^{13}$, P. Schillebeeckx ${ }^{29}$, S. Schmidt ${ }^{3}$, D. Schumann ${ }^{32}$, J.L. Taín ${ }^{16}$, G. Tagliente ${ }^{7}$,

A. Tsinganis ${ }^{9}$, S. Valenta ${ }^{9}$, G. Vannini ${ }^{25}$, V. Variale ${ }^{5}$, P. Vaz $^{13}$, A. Ventura ${ }^{26}$, R. Versaci $^{9}$,

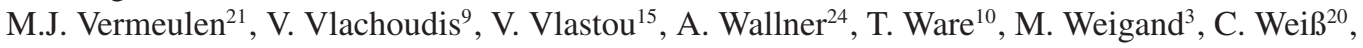

T. Wright ${ }^{10}$, and Zǔgec ${ }^{11}$

${ }^{1}$ Institut de Physique Nucléaire, CNRS, Unviersité Paris-Sud, 91406 Orsay, France

${ }^{2}$ Facultad de Fisica, Universidade de Santiago de Compostela, 15782, Spain

${ }^{3}$ Johann-Wolfgang-Goethe Universität, Frankfurt, Germany

${ }^{4}$ Uniwersytet Lódzki, Lodz, Poland

${ }^{5}$ Istituto Nazionale di Fisica Nucleare, Bari, Italy

${ }^{6}$ Centro de Investigaciones Energeticas Medioambientales y Tecnologicas (CIEMAT), Madrid, Spain

${ }^{7}$ Charles University, Prague, Czech Republic

${ }^{8}$ Commissariat à l'Energie Atomique (CEA) Saclay- Irfu, Gif-sur-Yvette, France

${ }^{9}$ European Organization for Nuclear Research (CERN), Geneva, Switzerland

${ }^{10}$ University of Manchester, Oxford Road, Manchester, UK

${ }^{11}$ Department of Physics, Faculty of Science, University of Zagreb, Croatia

${ }^{12}$ Universitat Politecnica de Catalunya, Barcelona, Spain

${ }^{13}$ Instituto Tecnologico e Nuclear, Instituto Superior Tecnico,Universidade Tecnica de Lisboa, Lisboa,

Portugal

${ }^{14}$ Universidad de Sevilla, Spain

${ }^{15}$ National Technical University of Athens (NTUA), Greece

${ }^{16}$ IFIC, Instituto de Fisica Corpuscular, CSIC-Universidad de Valencia, Spain

This is an Open Access article distributed under the terms of the Creative Commons Attribution License 2.0, which permits unrestricted use, distribution, and reproduction in any medium, provided the original work is properly cited. 
${ }^{17}$ Istituto Nazionale di Fisica Nucleare, Laboratori Nazionali di Legnaro, Italy

${ }^{18}$ Aristotle University of Thessaloniki, Thessaloniki, Greece

${ }^{19}$ Bhabha Atomic Research Centre (BARC), Mumbai, India

${ }^{20}$ Atominstitut, Technische Universitat Wien, Austria

${ }^{21}$ University of York, Heslington, York, UK

${ }^{22}$ Karlsruhe Institute of Technology, Campus Nord, Institut für Kernphysik, Karlsruhe, Germany

${ }^{23}$ Oak Ridge National Laboratory (ORNL), Oak Ridge, TN 37831, USA

${ }^{24}$ University of Vienna, Faculty of Physics, Vienna, Austria

${ }^{25}$ Dipartimento di Fisica, Universita di Bologna, and Sezione INFN di Bologna, Italy

${ }^{26}$ Agenzia nazionale per le nuove tecnologie, l'energia e lo sviluppo economico sostenibile (ENEA), Bologna, Italy

${ }^{27}$ Istituto Nazionale di Fisica Nucleare, Trieste, Italy

${ }^{28}$ Horia Hulubei National Institute of Physics and Nuclear Engineering - IFIN HH, Bucharest Magurele, Romania

${ }^{29}$ European Commission JRC, Institute for Reference Materials and Measurements, Retieseweg 111, 2440 Geel, Belgium

${ }^{30}$ Department of Physics and Astronomy, University of Basel, Basel, Switzerland

${ }^{31}$ Laboratori Nazionali del Gran Sasso dell'INFN, Assergi (AQ), Italy

${ }^{32}$ Paul Scherrer Institut, Villigen PSI, Switzerland

${ }^{33}$ Technical University of Munich, Munich, Germany

${ }^{34}$ Department of Physics and Astronomy, University of Basel, Basel, Switzerland

\begin{abstract}
Above $1 \mathrm{MeV}$ of incident neutron energy the fission fragment angular distribution (FFAD) has generally a strong anisotropic behavior due to the combination of the incident orbital momentum and the intrinsic spin of the fissioning nucleus. This effect has to be taken into account for the efficiency estimation of devices used for fission cross section measurements. In addition it bears information on the spin deposition mechanism and on the structure of transitional states. We designed and constructed a detection device, based on Parallel Plate Avalanche Counters (PPAC), for measuring the fission fragment angular distributions of several isotopes, in particular ${ }^{232} \mathrm{Th}$. The measurement has been performed at n_TOF at CERN taking advantage of the very broad energy spectrum of the neutron beam. Fission events were recognized by back to back detection in coincidence in two position-sensitive detectors surrounding the targets. The detection efficiency, depending mostly on the stopping of fission fragments in backings and electrodes, has been computed with a Geant 4 simulation and validated by the comparison to the measured case of ${ }^{235} \mathrm{U}$ below $3 \mathrm{keV}$ where the emission is isotropic. In the case of ${ }^{232} \mathrm{Th}$, the result is in good agreement with previous data below $10 \mathrm{MeV}$, with a good reproduction of the structures associated to vibrational states and the opening of second chance fission. In the $14 \mathrm{MeV}$ region our data are much more accurate than previous ones which are broadly scattered.
\end{abstract}

\title{
1. Introduction
}

A recent focus on the thorium cycle has been given for the future $4^{\text {th }}$ generation reactors. High accurate data are hence needed for the reactions involved in this cycle and several new projects started in this field of research to improve the existing nuclear data.

The n_TOF facility at CERN has this objective and mainly provides accurate cross sections for neutron-induced reactions. Fission fragment angular distributions (FFAD) is one of the interesting topics for data measurements, not only because they need to be taken into account in fission cross section measurements due to the effect of anisotropy on detection efficiency, but also because they give information on the spin and nuclear states of the fissioning system. Such information lead to a very good 

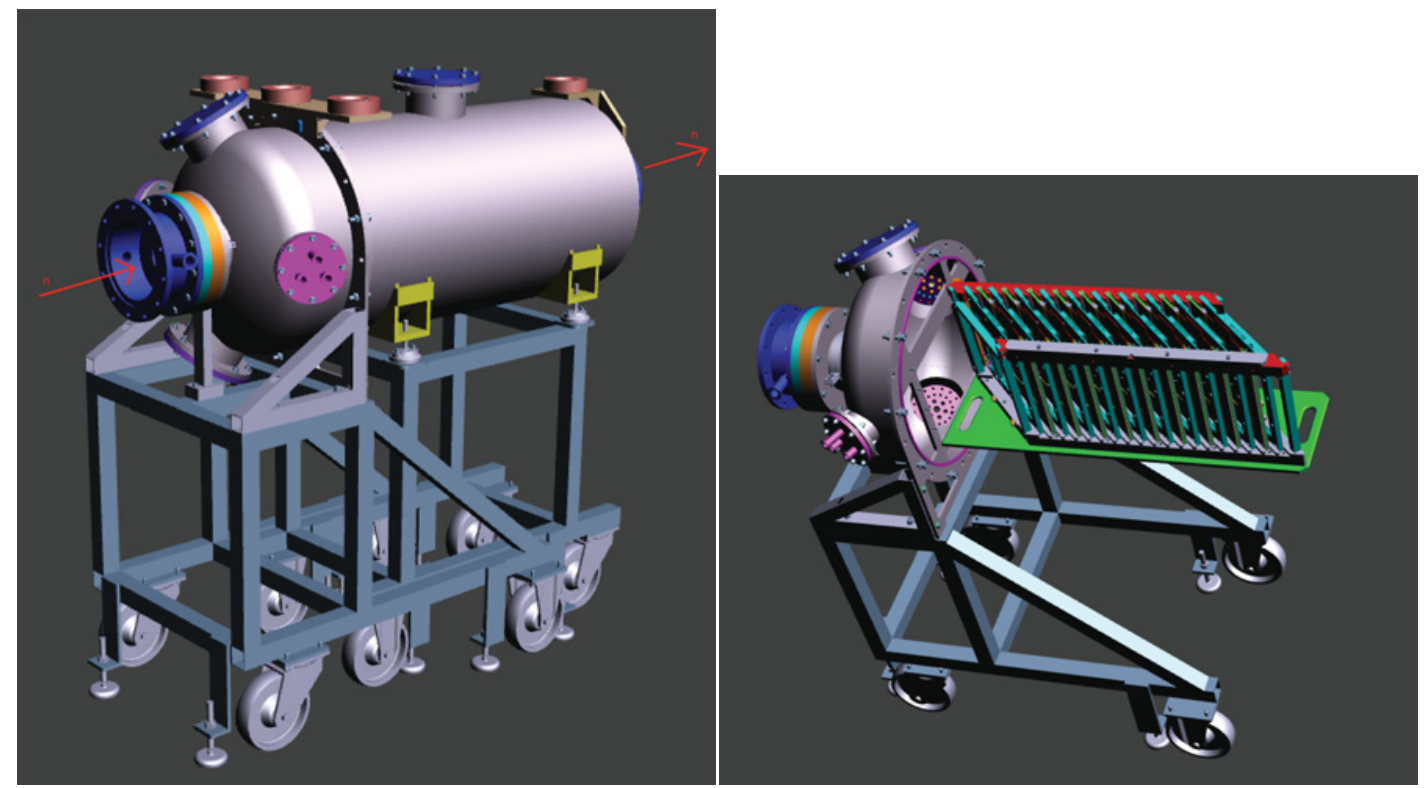

Figure 1. PPAC chamber, closed vessel (left), and detector and target arrangement (right).

understanding of the fission mechanism, and also of the momentum deposition in the reaction prior to fission, especially in the spallation domain.

\section{Experimental setup}

The neutron time-of-flight (n_TOF) facility [1] has been built at CERN in Geneva with the objective of studying neutron-induced reactions, especially radiative capture and fission, mainly for application to astrophysics, nuclear energy production and nuclear waste management.

The neutrons are produced by spallation reactions when $20 \mathrm{GeV}$ protons from the CERN Proton Synchrotron (PS) accelerator impinge onto a lead target. A very intense and pulsed neutron beam is generated and sent to the experimental room at $185 \mathrm{~m}$.

The measuring set-up is a chamber containing a stack of 10 Parallel Plate Avalanche Counters (PPACs) interleaved with 9 targets, as shown in in Figure 1, and the fission fragments emitted by each target are detected by the 2 PPACs bordering it. The neutron beam crosses all detectors and targets with a negligible attenuation due to the very thin targets and detectors.

The PPAC is a gaseous detector operating in proportional mode at low pressure. The chamber is entirely filled with $\mathrm{C}_{3} \mathrm{~F}_{8}$ at 4 mbar with a steady flow inside the detecting gaps.

For each detector the 3 electrode plates, 1 anode surrounded by 2 cathodes, are placed in a parallel configuration, $3.2 \mathrm{~mm}$ apart, and the cathodes are stripped in $\mathrm{X}$ and $\mathrm{Y}$ direction to provide a localization of the impact of the fission fragment track. Each strip is connected to a delay line having a total propagation time of $320 \mathrm{~ns}$. An impedance-matched charge pre-amplifier is connected to each end and the position of the avalanche is obtained by measuring the delay between the pulse on the anode and the pulses propagating along the delay lines.

The detectors and targets have been tilted by $45^{\circ}$ with respect to the neutron beam direction as shown in Figure 1, to get a full coverage of angles as explained in the next section. 


\section{Efficiency simulation}

Several actinides have been measured in three years, ${ }^{235} \mathrm{U},{ }^{234} \mathrm{U},{ }^{238} \mathrm{U},{ }^{237} \mathrm{~Np}$ and ${ }^{232} \mathrm{Th}$. The targets are produced by electrodeposition onto a thin aluminium foil of $2 \mu \mathrm{m}$ thickness for ${ }^{234} \mathrm{U},{ }^{235} \mathrm{U},{ }^{238} \mathrm{U}$ and ${ }^{237} \mathrm{~Np}$, and $0.75 \mu \mathrm{m}$ for ${ }^{232} \mathrm{Th}$. The active layer is a disk of $8 \mathrm{~cm}$ in diameter with a thickness ranging from 200 to $300 \mu \mathrm{g} / \mathrm{cm}^{2}$. With such thin samples and backings it is possible to detect the 2 fission fragments in coincidence on both sides of the target. The chemical compositions of the samples and backings for ${ }^{234} \mathrm{U},{ }^{235} \mathrm{U},{ }^{238} \mathrm{U},{ }^{237} \mathrm{~Np}$ have been well characterized by $\alpha$-spectroscopy, $\alpha$-counting, mass spectroscopy and Rutherford Backscattering Spectroscopy (RBS). ${ }^{235} \mathrm{U}$ is used as a reference because its fission cross section is accurately known up to $20 \mathrm{MeV}$ (uncertainty less than 1\%) and its FFAD is isotropic at low energy below $3 \mathrm{keV}$ because the incident angular momentum $(l=0$ or $l=1)$ is much lower than the unpolarized incident spin ( $S=3$ or 4$)$.

The measured angular distribution of detected fragments for ${ }^{235} \mathrm{U}$, as shown in Figure 2, it does not follow a flat distribution due to the detector efficiency which varies with $\cos \theta$, where $\theta$ is the angle against the beam. This efficiency is not defined by geometrical limitations, but it is related to the fission fragment slowing down in the aluminium foil of the backing and the detector electrodes, which may prevent the fission fragment from reaching the detecting gap. This is particularly noticeable when the angle $\cos \theta^{\prime}$ against the perpendicular to the layers and detectors increases because the sensed thickness goes as $1 / \cos \theta^{\prime}$.

Thus we used two methods to find this efficiency. The first one is a simulation based on the Geant 4 software[3] and the second is an experimental self-determination.

A simplified version of the set-up has been implemented with Geant4, using only two PPAC detectors and one target with a realistic description of geometry and materials: actinide layer, aluminium backing, mylar electrodes. The distance between target and each detector is $25 \mathrm{~mm}$. All materials are immersed in a $\mathrm{C}_{3} \mathrm{~F}_{8}$ gas at $4 \mathrm{mbar}$ as in the experiment. Two fission fragments are generated by selecting their charges and masses according to the ENDF/B-VII.1 library for ${ }^{235} \mathrm{U}$. Their kinetic energy are deduced from Viola's systematics [4], and the emission angle is assumed isotropic. As already mentioned this assumption holds for ${ }^{235} \mathrm{U}$ at low neutron energy, but is generally wrong for other isotopes and for energies greater than $1 \mathrm{MeV}$. However as long as we want to compute the dependence of the efficiency with $\cos \theta$ this assumption does not play any role, as it affects only the overall efficiency integrated over angles.

All the fragment tracks were followed until their disappearance either when stopped in the materials or when they escaped the set-up. Only the fission events where the 2 fragments penetrate all the detecting gaps were considered as detected.

The PPAC data analysis is based on the detection in coincidence of both fission fragments in two adjacent PPACs, allowing to reject most of the background produced by the $\alpha$ emission of the radioactive samples and by the other types of reaction above a few MeV. In order to obtain the emission angle we have used the cathode information which provides the fragment position in each detector. The combination of the information recorded on the two cathodes provides the $\mathrm{X}$ and $\mathrm{Y}$ positions. The angle of the fission track is reconstructed from the positions recorded in the two detectors on each side of the target, assuming a back to back emission at $180^{\circ}$. We computed the angles in the reference frame of the incident neutron $(\theta, \phi)$ and the angles relative to the perpendicular to the detectors $\left(\theta^{\prime}, \phi^{\prime}\right)$.

As mentioned before, below $3 \mathrm{keV}$, the FFAD of ${ }^{235} \mathrm{U}(\mathrm{n}, \mathrm{f})$ is known to be isotropic and the number of counts as a function of the $\cos \theta$ is exactly proportional to the detection efficiency.

In Figure 2, we show the agreement with the results obtained from a Geant 4 simulation and the experimental distribution. At $\cos \theta=1$ the efficiency is equal to 1 because this corresponds to $\cos \theta^{\prime}=0.7$ $\left(\theta^{\prime}=45^{\circ}\right)$ and the effective thickness crossed by the fission fragments is still low enough to allow their detection. At lower $\cos \theta$ lower values of $\cos \theta^{\prime}$ are reached depending of the $\phi$ angle and the efficiency 


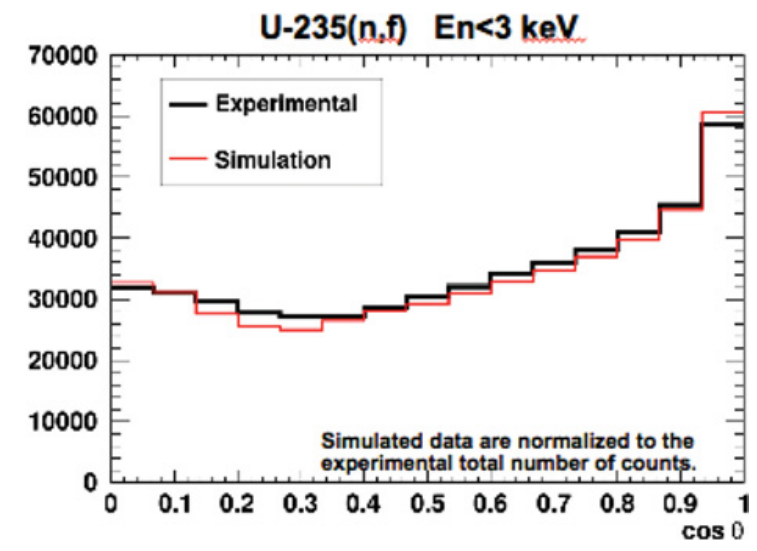

Figure 2. Comparison of simulated and measured detection efficiency for the isotropic emission of ${ }^{235} \mathrm{U}$.
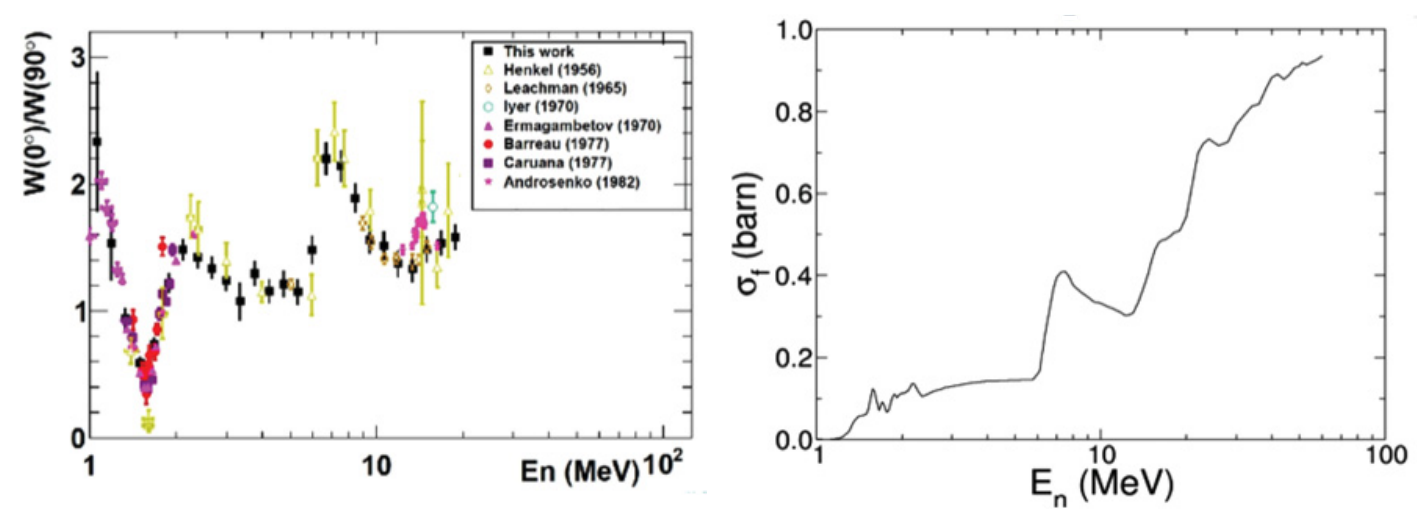

Figure 3. Left: the ${ }^{232} \mathrm{Th}$ anisotropy parameter as a function of the incident energy. Right: fission cross section on the same energy domain.

drops. The rise at low $\cos \theta$ results from the contribution of trajectories where the backward fragment is detected in the forward detector due to the tilting angle of $45^{\circ}$.

The conclusion of this comparison is that the simulation is able to predict accurately the angular dependence of the efficiency. Therefore we can use the simulation for each target, taking into account the proper backing thickness. The angular distribution will be obtained as the the angular histogram of counts divided by the simulated angular dependence of the efficiency.

\section{4. ${ }^{232}$ Th Results}

For every energy interval the angular distribution is fitted with even Lgendre polynomial up to order 4. The level of anisotropy is represented by the anisotropy parameter defined as $W\left(0^{\circ}\right) / \mathrm{W}\left(90^{\circ}\right)$ where $W(\theta)$ the value of the polynomial fit at $\theta$.

The energy dependence of the anisotropy parameter is shown in Figure 3 between fission threshold and $20 \mathrm{MeV}$.

First, the figure shows that below $10 \mathrm{MeV}$ the $\mathrm{n}_{-} \mathrm{TOF}$ data are consistent with the previous measurements [7-11] (extracted from EXFOR[5]). 


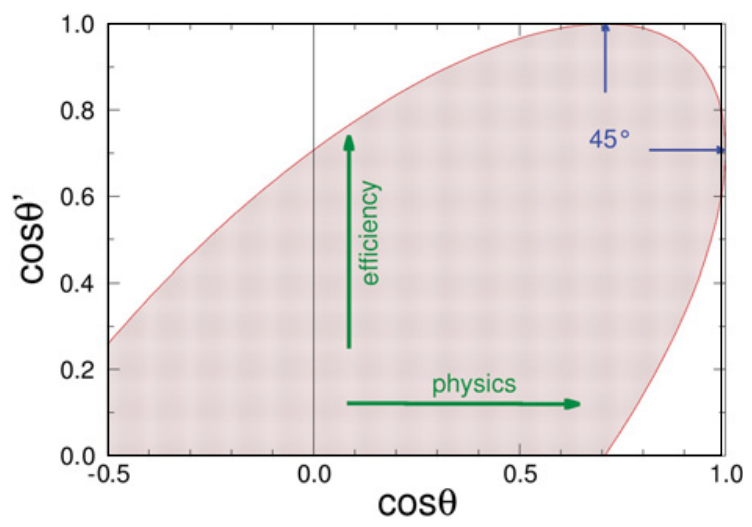

Figure 4. Available area for fission trajectories in the $\left(\cos \theta, \cos \theta^{\prime}\right)$ plane.

In particular the anisotropy exhibits a sudden dip around $1.6 \mathrm{MeV}$ which corresponds to fission through vibrational states, as already recognized in the past, and visible as structures in the fission cross section displayed in the right side. At this energy the distribution is sideward peaked around $90^{\circ}$ with respect to the beam direction. A steep rise of the anisotropy (forward-backward peaking) occurs at $6 \mathrm{MeV}$ where the second chance fission opens (fission after neutron emission), still in agreement with previous measurements.

At $14 \mathrm{MeV}$, in the vicinity of the third chance fission, the n_TOF data are definitely more accurate and draw a defined behavior where previous measurements were highly scattered.

\section{Self determination of the efficiency}

The efficiency correction used so far is based on simulations after having checked their validity on the ${ }^{235} \mathrm{U}$ target at low energy. However one may suspect that this computation is not always accurate because the experimental efficiency depends on some parameters which are not always known and may change from one target to the other: electronic thresholds, chemical composition of the target layer which affects the slowing down of the fragments, charge and mass distribution in fission which depends on the neutron energy. Therefore it would be highly desirable to get an experimental measurement of the efficiency, and it turns out that such a method can be implemented.

The method is based on the fact that the efficiency only depends on $\theta^{\prime}$ the angle against the normal to detectors and targets, because only this angle defines the effective thickness of the crossed layers which governs the slowing down of the fragments. The efficiency is equal to 1 for $\theta^{\prime}<\theta_{0}^{\prime}$ (with $\theta_{0}^{\prime}$ greater than $45^{\circ}$ ) and drops beyond this threshold corresponding to grazing trajectories on detectors for which some fragments are stopped in dead layers or release very low energy in the detecting gaps.

Conversely the angular distribution only depends on $\theta$ due to the cylindrical symmetry around the beam, in particular it doesn't depend on $\phi$ the azimuthal angle around the beam. For a given $\theta$ a range of $\theta^{\prime}$ is covered simply by sweeping $\phi$ over its domain $[-\pi, \pi]$. The available area in the map $\left(\cos \theta, \cos \theta^{\prime}\right)$ is shown in Figure 4. As $\cos \theta$ and and $\cos \theta^{\prime}$ are extracted experimentally the histogram of counts in the hatched area can be constructed.

Inside this available area a profile along a horizontal line, taking into account the solid angle of each cell, is simply affected by the angular distribution because the efficiency is constant along this line. On the contrary when following a vertical line ( $\theta$ constant) the variation of counts is merely proportional to the efficiency because all the points correspond to the same physical angle relative to the beam, with different $\phi$. As the efficiency is equal to 1 in the upper part of the diagram, it is possible to map the 
efficiency and the angular distribution by progressing down from the top to the bottom of the available elliptic area.

This method is presently under investigation and the results will be compared to those based on the simulation of the efficiency.

\section{Conclusion}

We developed and used a fission detection system based on PPAC and targets with very thin backings to measure the FFAD in neutron induced fission reaction of several isotopes. The efficiency correction has been implemented through a simulation and validated by comparison to the case of ${ }^{235} \mathrm{U}$ at low energy where the angular distribution is known to be isotropic. For ${ }^{232} \mathrm{Th}(\mathrm{n}, \mathrm{f})$ the structures appearing in the anisotropy are directly related to those in the fission cross section: vibrational resonances around $1.6 \mathrm{MeV}$, and openings of n-chance fission. From fission threshold to $10 \mathrm{MeV}$, we get a good agreement with previous data and at $14 \mathrm{MeV}$, in the vicinity of the opening of third chance fission, our data are much more accurate than previous ones. We are exploring a self-determination method to extract directly the efficiency from the measurement and we will compare it to the simulation method.

\section{References}

[1] U. A. et al. (The n TOF Collaboration), Measurements of Fission Cross Sections for the Isotopes relevant to the Thorium Fuel Cycle, CERN/INTC 2001-025 (2001)

[2] M. B. Chadwick, M. Herman, P. Oblozinsky, et al., ENDF/BVII.1: Nuclear data for science and technology: Cross sections, covariances, fission product yields and decay data, Nuclear Data Sheets 112 (2011) 2887

[3] S. Agostinelli, J. Allison, K. Amako, et al., Nucl. Instr. and Meth. A 506 (2003) 250. Geant4 official webpage: http://geant4.web.cern.ch/geant4/

[4] V. E. Viola, K. Kwiatkowski, M. Walker, Phys. Rev. C 31 (1985) 1550

[5] Viktor Zerkin, NDS, International Atomic Energy Agency, http://www-nds.iaea.org/ exfor/exfor.htm

[6] D. Tarrio, Neutron-induced fission fragment angular distribution at CERN n TOF: The Th-232 case, Ph.D. thesis, Universidade de Santiago de Compostela, Spain, 2012

[7] R. L. Henkel, J. E. B. Jr., Phys. Rev. 103 (1956) 1292

[8] S. B. Ermagambetov, G. N. Smirenkin, Sov. J. Nucl. Phys. 11 (1970) 646

[9] G. Barreau, C. E. N. Bordeaux-Gradignan Reports 7706 (1977)

[10] J. Caruana, J. W. Boldeman, R. L. Walsh, Nucl. Phys. A 285 (1977) 205

[11] B. M. Gokhberg, L. D. Kozlov, S. K. Lisin, et al., Sov. J. Nucl. Phys. 47 (1988) 201 